\title{
Gamma-ray observations of binary systems with H.E.S.S.
}

Pol Bordas (for the H.E.S.S. Collaboration) ${ }^{* \dagger}$

Institut für Astronomie und Astrophysik Tübingen

E-mail: pol.bordas@uni-tuebingen.de

We report on recent observations of binary systems obtained with the High Energy Stereoscopic System of Cherenkov telescopes. High and Very High Energy emission from a few binaries has been detected by both space and ground-based observatories in recent years. These data are providing crucial information on the physics of relativistic outflows and the engines powering them. Yet, many questions remain open, for which further data are required. New H.E.S.S. observations of the systems HESS J0632+057, PSR B1959-63/LS 2883, and the recently discovered source HESS J1018-589 are briefly summarized, as well as the search for TeV emission from new candidates, including the colliding-winds binary system Eta Carinae. Our results confirm the predictions derived previously for some of those sources, but unexpected results are also found in a few cases, which are discussed in the context of contemporaneous observations covering other energy bands.

"An INTEGRAL view of the high-energy sky (the first 10 years)" 9th INTEGRAL Workshop and celebration of the 10th anniversary of the launch,

October 15-19, 2012

Bibliotheque Nationale de France, Paris, France

\footnotetext{
* Speaker.

${ }^{\dagger}$ The support of the Namibian authorities and of the University of Namibia in facilitating the construction and operation of H.E.S.S. is gratefully acknowledged, as is the support by the German Ministry for Education and Research (BMBF), the Max Planck Society, the French Ministry for Research, the CNRS-IN2P3 and the Astroparticle Interdisciplinary Programme of the CNRS, the UK Science and Technology Facilities Council (STFC), the IPNP of the Charles University, the Polish Ministry of Science and Higher Education, the South African Department of Science and Technology and National Research Foundation, and by the University of Namibia.We appreciate the excellent work of the technical support staff in Berlin, Durham, Hamburg, Heidelberg, Palaiseau, Paris, Saclay, and in Namibia in the construction and operation of the equipment. This research has made use of the NASA/IPAC Infrared Science Archive, which is operated by the Jet Propulsion Laboratory, California Institute of Technology, under contract with the National Aeronautics and Space Administration. PB has been supported by grant DLR 50 OG 0601 during this work. PB also acknowledges the excellent work conditions at the INTEGRAL Science Data Center.
} 


\section{Introduction}

Gamma-ray binary systems are a new class of High- and Very High Energy (HE, $100 \mathrm{MeV} \leq$ $E \leq 100 \mathrm{GeV}$; VHE, $E>100 \mathrm{GeV}$ ) gamma-ray emitters. They are characterized by a high-level flux observed at $\mathrm{MeV}-\mathrm{GeV}$ energies, displaying also variable and in most cases orbitally modulated emission. Only a few systems have been discovered so far. All of them are high-mass X-ray binaries, consisting of a compact object orbiting around a massive star of $\mathrm{O}$ or Be type. The presence of a neutron star has been clearly identified only in the gamma-ray binary PSR B195963/LS 2883 [1], whereas for the systems LS 5039 [2], LS I +61 303 [3, 4] and the recently discovered sources HESS J0632+057 [5, 6] and 1FGL J1018.6-5856 [7], the nature of the compact object is as yet unknown. A few more binaries have been recently detected at HE energies, namely Eta Carinae [8, 9], Cyg X-3 [10, 11] and Cyg X-1 [12], but their counterparts at VHE are still lacking or unconfirmed (see, e.g., [13, 14, 15]).

We report here a summary of the recent H.E.S.S. observations of the systems PSR B1959-63/LS 2883, HESS J0632+057, HESS J1018-589 (with its possible association to 1FGL J1018.6-5856) and Eta Carinae. A complete description of the observations, the data-analysis performed and the obtained results can be found in [16, 17, 18] and [15], respectively.

\section{Observations}

\subsection{PSR B1959-63/LS 2883}

The gamma-ray binary system PSR B1959-63/LS 2883, originally discovered in a high-frequency radio survey devoted to the detection of young and short-period pulsars [19], consists of a rapidly rotating neutron star with a spin period of about $48 \mathrm{~ms}$ and a spin-down luminosity $\sim 10^{35} \mathrm{erg} \mathrm{s}^{-1}$ orbiting around a massive Be star every $\sim 3.4$ years in a highly eccentric $(e=0.87)$ orbit . The dense equatorial disk from the companion star is inclined with respect to the pulsar orbital plane [20] such that the pulsar crosses the disk twice in each orbit. Since its discovery, PSR B195963/LS 2883 has been studied at all energy bands. The system was observed by H.E.S.S. in the 2004 and 2007 periastron passages, at slightly different orbital phases, providing a significant detection at VHEs on both occasions [2, 21].

New H.E.S.S. observations were scheduled to cover the 2010/2011 periastron passage. However, in this occasion the source was not visible for H.E.S.S. before and at the periastron passage. In addition, observations were partially hampered by bad weather conditions, resulting in a total data-set of $\sim 6 \mathrm{~h}$ of livetime after the quality selection cuts [16]. These new observations partially overlapped with the start time of a strong gamma-ray flare reported by the Fermi-LAT, about $30 \mathrm{~d}$ after periastron. This HE flare lasted for about 7 weeks, displaying an average flux $\sim 10$ times higher than that close to the periastron, and implied a very high efficiency for the conversion of the pulsar rotational energy into gamma-rays, approaching a 100\% level [22].

The new H.E.S.S. observations resulted in a clear detection of PSR B1959-63/LS 2883, at an $\sim 11.5 \sigma$ significance level, and both the flux level and the spectral properties are consistent with those obtained in previous periastron observations [16]. No signature of the emission enhancement seen at $\mathrm{GeV}$ energies is observed at VHEs. A careful statistical study based on the Fermi and 
H.E.S.S. lightcurves has been performed to check for this different behavior using the three-day overlap of the HE and VHE observations. Both the H.E.S.S. and Fermi data are divided into "preflare" and "flare" intervals. The HE flux ratio $\phi_{\text {flare }}^{\mathrm{HE}} / \phi_{\text {pre-flare }}^{\mathrm{HE}} \geq 9.2$ in the Fermi data, whereas a profile likelihood method applied to the VHE data yields a value $\phi_{\text {flare }}^{\mathrm{VHE}} / \phi_{\text {pre-flare }}^{\mathrm{VHE}}<3.5(99.7 \%$ C.L.). The $\mathrm{GeV}$ and $\mathrm{TeV}$ emission may have therefore a different origin; if they were produced by the same processes, a flux enhancement of a similar magnitude in both energy bands would be expected. This conclusion is further supported by a joint fit to the HE and VHE spectra [16], which can not account for both emissions with any reasonable model, in particular when accounting for the reported Fermi-LAT upper limits at energies 1-100 GeV. One possible intrepretation could be that VHE emission is produced by the shocked pulsar wind particles, the same responsible for the radio and X-ray emission, whereas the GeV flare could correspond to IC by the unshocked relativistic wind scattering off the companion's photon field as well as photons from a heated part of the Be circumstellar disk [23].

\subsection{HESS J0632+057}

The point-like TeV source HESS J0632+057 was discovered by H.E.S.S. during the Galactic Scan program [5] and later confirmed by further VERITAS ([24, 25]) and MAGIC ([26]) observations. Its position is coincident with the massive B0pe-type star MWC 148 (HD 259440), for which optical spectroscopic observations have been recently obtained and confirm that HESS J0632+057 is indeed a binary system ([27]; see also [31]). HESS J0632+057 is also coincident with the ROSAT source 1RXS J063258.3+054857, the unidentified gamma-ray source 3EG J0634+0521, and has a point-like radio counterpart coincident with MWC 148 [28], which also shows variable extended radio emission [29]. Its radio and X-ray properties [6, 30, 31, 32] resemble those found in LS 5039, LS I +61 303 and PSR B1959-63, although further studies are required to assess if a common scenario is at work in all cases.

The total H.E.S.S. data-set on HESS J0632+057 spans a wide time interval from 2004 to 2012, amounting to $\sim 47 \mathrm{~h}$ of observations. Such a long time-range implies a rather variable observing conditions, in particular for the pointing zenith angle, which spans from about $28^{\circ}$ to $58^{\circ}$, with an average of $34.2^{\circ}$. The latest H.E.S.S. data correspond to the 2011/2012 campaign, with observations taken in December 2011 and February 2012, which provide a total of $8.2 \mathrm{~h}$ of data after standard quality cuts filtering. However, the expected maximum could not be well-sampled in this occasion due to bad weather conditions, and most of the observations fell in the so-called X-ray "dip" orbital phase range right after the maximum ([31, 17]). The total data-set also includes H.E.S.S. observations corresponding to orbital phases unexplored so far by any current Cherenkov telescope, in particular at phases in the range [0.7 - 0.8], which were taken in March 2007, January 2008 and October 2009 and amounts to $\sim 7 \mathrm{~h}$ of observing time. A preliminary analysis making use of the Model analysis technique [33] provides a $4.1 \sigma$ significance detection at those phases. A cross-check of these results, performed using a Hillas based analysis for the same run list, which also makes use of an independent calibration of the raw data, provides compatible results. A detailed report on these results, including an updated H.E.S.S data-set together with VERITAS and Swift-XRT long-term observations is in preparation. 


\subsection{HESS J1018-589}

The Fermi-LAT collaboration has recently reported on the detection of the new gamma-ray binary candidate 1FGL J1018.6-5856 [35]. The periodic modulation of $P_{\text {orb }}=16.58 \pm 0.02$ days observed in the Fermi-LAT data strongly suggests a binary nature of the source, with the O6Vtype star 2MASS 10185560-5856459 proposed as the companion star [35]. Further indications of the its binary nature are provided by the observation of periodic X-ray and radio emission from a point-like source spatially coincident with 1FGL J1018.6-5856 [35]. At hard X-ray wavelengths, Li et al. (2011) [36] found a possible INTEGRAL counterpart to the system, with also a hint of anticorrelation with respect to the Fermi-LAT phase-folded lightcurve.

H.E.S.S. pointed towards the region around 1FGL J1018.6-5856 mainly during a multi-year observation campaign in 2007, 2008 and 2009 on Westerlund 2, leading to the detection of two distinct emission regions [18]. The first one consists of a diffuse emission region that extends towards the direction of PSR J1016-5857, the position of which is compatible with that of the pulsar, whilst the second one is a point-like source at the center of G284.3-1.8, which is spatially compatible with 1FGL J1018.6-5856. No flux variability could be derived from the H.E.S.S. data, and the LombScargle test did not recover the $16.58 \mathrm{~d}$ orbital period previously identified [35]. Furthermore, it is not clear yet if the extended nearby source could be affecting the point-like source analysis. Finally, an inadequate sampling of the binary orbital phases could partially explain the lack of detection of the expected modulation. Dedicated VHE observations will help to properly establish the possible source variability and/or periodicity, clarifying its association with 1FGL J1018.6-5856.

\subsection{Eta Carinae}

Eta Carinae is a binary system located in the Carina Nebula, one of the most active HII region in our Galaxy. The system is composed of a Luminous Blue Variable (LBV) and an O- or Btype companion star, orbiting one another with a period of $\sim 5.5$ years in a highly eccentric orbit (e 0.9). The LBV displays a strong mass loss rate, $\dot{M} \geq 5 \times 10^{-4} M_{\odot} \mathrm{yr}^{-1}$, with a terminal wind velocity of $v_{\text {wind }} \sim(500-700) \mathrm{km} \mathrm{s}^{-1}$. Together with the (less exteme) wind parameters of the companion star, the system outputs a total wind kinetic energy of about $10^{37} \mathrm{erg} \mathrm{s}^{-1}$ (see, e.g. [37] and references therein). The collision region of the stellar winds may lead to efficient particle acceleration [38], which could be behind the high-energy emission observed from the system in Xrays [39, 40] and HE gamma-rays [41, 42]. Recently, a variable high-energy component has been found in the Fermi-LAT data, extending up to $\gtrsim 100 \mathrm{GeV}$ [9, 43], further motivating the search for a possible VHE signal from the source.

H.E.S.S. observed the Carina region between 2004 and 2010 for a total of 33 hours after standard quality selection cuts (see [15] for details). Observations were taken at zenith angles in the range $36^{\circ}$ to $54^{\circ}$, with a mean value of $39^{\circ}$. The average pointing offset from the target position was $0.8^{\circ}$. Data were analysed with the H.E.S.S. Standard Analysis software and made use of a Hillasbased Boosted Decision Trees method for an efficient suppression of the hadronic background component [34] (see [15] for details). No significant signals of VHE $\gamma$-ray emission from Eta Carinae or the Carina Nebula have been obtained from the H.E.S.S. observations. A $99 \%$ confidence level upper limit on the integral $\gamma$-ray flux above $470 \mathrm{GeV}$ at the level of $7.7 \times 10^{-13} \mathrm{ph} \mathrm{cm}^{-2} \mathrm{~s}^{-1}$ and $4.2 \times 10^{-12} \mathrm{ph} \mathrm{cm}^{-2} \mathrm{~s}^{-1}$ have been derived for the central system and the surrounding nebula, 
respectively. The non-detection, together with the spectral properties of the high-energy component observed in the Fermi-LAT data, implies the presence of a cut-off at energies $\lesssim 1 \mathrm{TeV}$, caused either by a cut-off in the accelerated particle spectrum or as a consequence of a strong $\gamma-\gamma$ absorption in the inner regions of the binary system in either leptonic or hadronic scenarios (see [9, 44]).

\section{Concluding remarks}

H.E.S.S. observations are providing new and exciting insights on most of the currently known gamma-ray binaries. Our latest results unambiguously disentangle the HE and VHE emission in the pulsar-wind/disk interaction region in PSR B1259-63/LS 2883 during a recently detected HE flare. They reveal a periodic detection at VHE of HESS J0632+057 at orbital phases close to the maximum of the X-ray lightcurve, whereas also a hint of emission at unexpected orbital phases is observed. They suggest a VHE counterpart within HESS J1018-589 of the recently discovered gamma-ray binary 1FGL J1018.6-5856. Finally, upper limits obtained at VHEs on Eta Carinae, together with the Fermi-LAT detection of a variable high-energy component in the source spectrum, imply the presence of a cut-off in the particle energy distribution and/or the signatures of strong attenuation due to $\gamma-\gamma$ absorption close to the wind-wind collision region.

Further H.E.S.S. observations of binary systems are foreseen in the near future. They will make use of the recent H.E.S.S. II upgrade, providing an improved sensitivity and, for the first time, the possibility to use the Cherenkov technique to detect sources down to $\sim 30 \mathrm{GeV}$ energy thresholds in comparatively short $(\sim$ few $\times 10 \mathrm{~h})$ exposure times.

\section{References}

[1] Aharonian, F. et al. (HESS Collaboration) 2005a, Science, 309, 746

[2] Aharonian, F. et al. (HESS Collaboration) 2005b, A\&A, 442, 1

[3] Albert et al. (MAGIC Collaboration) 2009, ApJ, 693, 303

[4] Acciari et al. (VERITAS Collaboration) 2011, ApJ, 738, 3

[5] Aharonian, F. et al. (HESS Collaboration) 2007, A\&A, 469, L1

[6] Hinton, J., Skilton, J. L., Funk, S. et al. 2009, ApJ, 690, L101

[7] Fermi-LAT Collaboration 2012, Science, 335, 189

[8] Abdo A. A. et al. (Fermi-LAT collaboration), 2009a, ApJS, 183, 46

[9] Farnier C., Walter R. \& Leyder J.C. 2011, A\&A, 526, A57

[10] Abdo A. A. et al. (Fermi Collaboration) 2009b, Science, 326, 1512

[11] Tavani, M., Bulgarelli, A., Piano, G. et al. 2009a, Nature, 462620

[12] Sabatini, S., Tavani, M., Striani, E. et al. 2010, ApJ, 712, 10S

[13] Albert et al. (MAGIC Collaboration) 2007, ApJ, 665, 51

[14] Aleksic̀, J., Antonelli, L. A., Antoranz, P. et al. 2010, ApJ, 721, 843 
[15] H.E.S.S. Collaboration 2012b, MNRAS,424, 128

[16] H.E.S.S. Collaboration 2013, accepted for publication in A\&A (arXiv:1301.3930)

[17] Bordas, P. (for the H.E.S.S. Collaboration) \& Maier, G. (for the VERITAS Collaboration) 2012 , AIPC, 1505, 366

[18] H.E.S.S. Collaboration 2012a, A\&A, 541A, 5

[19] Johnston, S., Manchester, R. N., Lyne, A. G. et al. 1992b, ApJ, 387, L37

[20] Negueruela, I., Ribò, M., Herrero, A. et al. 2011, ApJ, 732, L11

[21] Aharonian, F. et al. (HESS Collaboration) 2009, A\&A, 507, 389

[22] Abdo A. A. et al. (Fermi-LAT collaboration), 2011, ApJ, 736L, 11

[23] Khangulyan, D., Aharonian, F. A., Bogovalov, S. V. \& Ribó, M. 2012, ApJ, 752, 17

[24] Acciari, V. A. et al. (VERITAS collaboration), 2009, ApJ, 698, 94

[25] Ong, R. et al. (VERITAS collaboration) 2011, The Astronomer's Telegram 3153

[26] Aleksić et al. (MAGIC Collaboration) 2012, ApJ, 754L, 10

[27] Casares, J., Ribó, M., Ribas, I. et al. 2012, MNRAS, 421, 1103

[28] Skilton, J. L., Pandey-Pommier, M., Hinton, J. A. et al. 2009, MNRAS, 399, 317

[29] Moldón, J., Ribó, M. \& Paredes, J. M. 2011, A\&A, 533, L7

[30] Falcone, A. D., Grube, J., Hinton et al. 2010, ApJ, 708, 52

[31] Bongiorno, S. D., Falcone, A. D., Stroh M. et al. 2011, ApJ, 737, L11

[32] Rea, N. \& Torres, D. F. 2011, ApJ, 737, L12

[33] de Naurois, M. \& Rolland, L. 2009, APh, 32, 231

[34] Ohm, S., van Eldik, C. \& Egberts, K. 2009, APh, 31, 383

[35] Ackermann, M. et al. (Fermi Collaboration) 2012, Science, 335, 189

[36] Li, J., Torres, D. F., Chen, Y. et al. 2011, ApJ, 738, 31

[37] Pittard J. M. \& Corcoran, M. F. 2002, A\&A, 383, 636

[38] Reimer, A., Pohl, M. \& Reimer, O. 2006, ApJ, 644, 1118

[39] Sekiguchi, A., Tsujimoto, M., Kitamoto, S. et al. 2009, PASJ, 61, 629

[40] Leyder, J., Walter, R. \& Rauw, G., 2010, A\&A, 524, 59

[41] Tavani, M., Sabatini, S., Pian, E. et al. 2009b, ApJ, 698, 142

[42] Abdo A. A. et al. (Fermi Collaboration) 2010, ApJ, 723, 649

[43] K. Reitberger, K., Reimer, O., Reimer, A. et al. 2012, A\&A, 544, 98

[44] Bednarek W. \& Pabich J. 2011, A\&A, 530, A49 